\title{
Progress in Research Contents and Methods of Theoretical Toxicology
}

\author{
Yunshen Jiang \\ Department of Toxicology, Nanjing Medical University, Nanjing, China \\ Email address: \\ yujdx@sina.cn
}

\section{To cite this article:}

Yunshen Jiang. Progress in Research Contents and Methods of Theoretical Toxicology. International Journal of Clinical and Experimental Medical Sciences. Vol. 5, No. 1, 2019, pp. 10-13. doi: 10.11648/j.ijcems.20190501.13

Received: February 24, 2019; Accepted: April 2, 2019; Published: April 22, 2019

\begin{abstract}
Toxicology is a modern science. Because of the rapid development of industry and agriculture, the amount of chemicals increases explosively. There has been more than 26,000,000 chemicals registered in the world and over 2000 new chemicals are produced every year. Therefore the traditional methods of toxicological evaluation cannot meet the requirements in the modern society, let alone the precise toxicological study for various chemicals. It is needed to make innovation in theory, so as to shorten the period, reduce the expenditure and input in the research, which may make the applications of chemicals safer. Meanwhile, as animal welfare is putting on the public agenda, less animals will be permitted to be used largely in the toxicological research. All of these promote the development of theoretical toxicology.
\end{abstract}

Keywords: Theoretical Toxicology, Big Data Processing, Cloud Computing

\section{Introduction}

Toxicology can be categorized into two subject areas, the theoretical toxicology and the experimental toxicology. In the early phases of development of toxicology, animal tests on carcinogenicity \& teratogenicity \& mutagenicity and acute/subacute/chronic toxicity were widely used. With the rapid development of industry and agriculture in recent years, the number of chemicals requiring toxicological analysis shoots up, for which the traditional testing pipelines would not satisfy. In the 1950s and 1960s, toxicology has not been formed as an independent subject in China. Through a half century's efforts, researchers accumulated a large amount of data. Along with the development of informative/computational/economical toxicology, especially big data processing and cloud computing, contributing a lot to the establishment of theoretical toxicology [1-4].

\section{Research Connotations}

Philosophy of toxicology. Medical philosophy is the philosophy of general essence and law of universal phenomenon in medical science. In 1977, G. L. Engel raised bio-psycho-social medical model for dissolving health and disease issues in human beings. This model and philosophy played an important role in development of toxicology. The philosophy of toxicology was based on medical philosophy. Toxicology is the study of mechanism and damage on organism and ecosystem of exogenous chemical, physical and biological factors and helps to prevent and cure the damage from these adverse factors exposure. The animal experiments (integral experiments) are the major ways in toxicology research, and huge amount of animals were sacrificed every year,, so ethically treatment of animals and ethic review should be essentiallycarry out on animal experiments. In order to better implement the animal $3 \mathrm{R}$ principle, other toxicological experimental methods should be performed, including the theoretical toxicology.

Establish a comprehensive understanding of the nature of human beings from a medical perspective, such as the concept of life in which biological attributes, social attributes, and thinking attributes are unified, that is, the philosophical thought of the unity of nature and man. The World Health Organization (WHO) has recently proposed a definition of health: physical health, mental health, social adaptation health and morality health. Traditional health definitions only include physical health and mental health. In the future, definition and instructions of health would be 
fixed more perfectly.

Covering the fundamental viewpoints about the nature and general laws of human health and disease, namely the concept of human body, health and disease.

Covering medical epistemology, empirical epistemology, logical thinking and non- logical thinking.

Following the development of toxicology methodology. In the past years, the incidence rate and mortality were the end point observation indicators. With the development of science and technology, the observation indicators of toxicology have gradually increased from poisoning to subhealth, health and longevity. Also, the research method has gradually transformed from structural toxicology to functional toxicology. On the other hand, the research methods have evolved from pathological research to biochemical, physiological and molecular biology research, which are becoming more and more refined. Medical research is moving from the cellular level to the electronic particle level. The emergence of quantum medicine can proactively detect the manifestations of microscopic states, making the prevention and detection of diseases much more detail and precise, eliminating the so-called philosophical debate in the medical field. In 1992, the famous toxicologist Terhard Zhinden at the 6th International Conference on Toxicology pointed out that experimental toxicology research is divided into three eras: i) the era of discovery; ii) the era of biological mechanism exploration; iii) the era of individual expression. However, theoretical toxicology has not been elaborated. In the past, theoretical toxicology was not specifically classified and was included only in general toxicology books. In fact, theoretical toxicology also has different branches, such as toxicology history, philosophy, bioinformatics and computational toxicology. Predecessors have began their research instudying the quantitative structure-activity relationship (QSAR) for a long time. For example, the former Soviet toxicologists used the formula to calculate the maximum allowable concentration (MAC). Chinese toxicologist Gu Zuwei also used formula to calculate the toxicity of pesticides. Toxicology center rule, doseresponse relationships, etc., have been used to predict and extrapolate toxicity to harmful factors such as chemicals, drugs, and physics. It is only that the above studies have not been explicitly attributed to theoretical toxicology. We gradually clarify the difference between microscopic state and macro-medical model step by step, gradually combine microscopic state particles with macroscopic symptoms. The application of microcosm theory was also used in ecotoxicology research. The risk assessment model was also established in evaluation system. 2.5 Applications on big data processing is the efficiency revolution on theoretical toxicology, for example, the introduction of structure effect mechanism and cost-effective mechanism. In theinformation and intelligence time, the big data processing is much more better and useful to process four $\mathrm{V}$, volume, velocity, variety and veracity in analysis of toxicological studies. For example, reactions between chemical structures and effects can be divided into three parts, teratology, carcinogenicity and hematological system damage. Recently the United Union cooperated with Baidu, setting up the big data processing laboratory in assistance of environment and health issues. Another example is the evaluation of manufacturing cost and application of chemicals in economic toxicology. There can be different evaluation system and reports from different companies with different methods on toxicity identification, sothe computational processing on these data is essential and in great need.2.6 Cloud computing service is the cheap internet service dynamically provided in requirement of our need. It leverages resources distributed across a large pool of computing resources to enable applications to acquire computing power, storage spaceand information services as needed. In the future, italso required a large amount of network information to serve the Toxicology, saving time and cost and obtaining information in a timely manner.2.7 Introduce new ideas, theories, methods or technologies in toxicology, and promote toxicology research to a new higher level in the future [5-7].

\section{Methods and Key Points}

Studies on medicine philosophy is the broader trend keeping pace with the times. In a climate of ever-changing concept of health, our thinking on studies of medicine philosophy also needs continuously updating. Toxicology studies gradually transform into function studies from structure studies. Morbidity and mortality were taken as observed indexes before toxicology, for which carcinogenicity, teratogenicity and mutagenicity were all structural changes. Nowadays, we could diagnose diseases much earlier and better with bio-photonic observations of functional changes in proper blend of microscopic particles and macro symptoms.

Study the mode of philosophy thinking of quantum theory and understand toxicology from the perspective of system theory instead of mechanistic theory. Materials can be a singular composite, which cannot be divided or broken down into other substances, or a complex system composed of several subsystems. There are frequent matter and energy exchange with the environment, which is the solid foundation of the existence of system. The quantum theory indicates that materials and their movements are discrete and discontinuous.

Studies on informatics are also very important. It is the time of information and intelligence. The development of theoretical toxicology needs to stand on the bleeding edge of itself and in the frontier of experimental toxicology and direct the development of experimental toxicology. Researchers should introduce more new theories and methods in toxicology and frontier concepts, theory, methods and technologies of other subjects, such as biology, chemistry, physics and pharmacy, etc. to guide the toxicology studies. What is more, it is highly necessary to draw experience and lessons from previous studies and avoid detours in the future.

The application of computational toxicology may play an 
important role in the future. In our country, we made great progress on the toxicity prediction, algorithm and model specification of environmental chemicals. Toxicologists fused toxicology, modern computer and information technology, chemistry and molecular biology into computational toxicology and predictive toxicology. These new branches of toxicology helped a lot in prediction of toxicity of new chemicals and guidance the developments of new products. On the other hand, to meet the demand of new product developments and fast safety evaluation, researchers proposed discovery toxicology and evidence-based toxicology. These new branches and theories, taken together, have directive significance to toxicological studies.

Establish chemical management and safety evaluation system with Chinese characteristics in line with international practice. Management toxicology is also a hot area of research focus in theoretical toxicology.

It is essential to reconsider the fields of priority in theoretical toxicology according to our national situation and raise the prevention and pollution control with multiple levels, aspects and targets of studies. Theoretical toxicologists must provide powerful, reasonable, lawful and practical management ordinance for legislation and regulation of public health in our country. There are tight connections between molecular biology and theoretical toxicology. So new methods and techniques of transgenic lines and mutants of experimental animals should be introduced in time for illustration of important mechanism of chemical toxicity. Some frontier technologies, such as systematic analysis, RNA-seq, DNA chips and microarray can be used to detect the up-regulation and down-regulation, trap and variance analysis of expression of thousands of genes at the same time. They offered great help on studies of mechanism on teratogenesis. The gene map on chromosomes would be used in distinguish of specific and non-specific molecular and cell damage. Also, some biomarkers were widely used in toxicological studies. For example, the nuclear magnetic resonance was used to analysis the metabolites in urine to study and understand more on metabolic pattern of toxic reaction biomarkers. Additionally, more technologies will be applied on evaluation of toxicity in the future. From integral experiment to substitution experiment with replacement, reduction and refinement rules, it is the development direction of experimental toxicology. And the direction of theoretical toxicology would contribute much more on studies of experimental toxicology. More new theories would also be put forward and improve the development of scientific practices [7-11].

\section{Prospects}

Toxicology cannot develop if isolation from theoretical toxicology. So, the study of theoretical toxicology and communications among colleagues need strengthening. To make few detours, the government departments should unify leadership and avoid repetition and waste on single chemical. As for experimental conditions, tests on chemicals would be much more comparable under unified methods and conditions with specified experimental animals [12-15].

\section{Conclusions}

In the new era, theoretical toxicology has endowed new concepts and contents, especially the application of technologies and methods of big data, informatics and cloud computing services, which has greatly promoted the development of theoretical toxicology. In addition, through the combination of medical science and quantum theory philosophy, theoretical toxicology can better guide the scientific application in toxic assessment, environmental health hazards and corresponding policy formulation and management.Theoretical toxicology has broad prospects for development.

\section{References}

[1] Zuang shi-xion. The development and prospect of toxicology in China. Chinese journal of preventive medicine 2008, 42 (s):9-10.

[2] Jiang yun-shen, Zhou jian-wei. Future toxicology---Quantum toxicology Chin J ind hyg occup dis 2014, 32(11)10-12.

[3] Wang xing. Big data analysis: methods and applications.. Beijing Tsinghua university press 2013, 1-4.

[4] Wang peng: Approaching cloud computing People's post and telecommunications publishing house Beijing 2009:1-2.

[5] Peleg Y, Pnini R, Zaaruru E. et al. Quantum mechanics.. Schuam's outline series 2nd. [M]. New York. Mcgrew hill company, 2010:21-40.

[6] Kanno J,: Reverse toxicology as a future predictive toxicology [M] Inoue $\mathrm{T}$, Rennie $\mathrm{W}$ D:Toxicologenomics. Tokyo: Springer-verlag. Tokyo. Inc. 2003:213-218.

[7] Zhu yon-liang, Ye zu-guang: Advance in computational toxicology and prediction of toxicity of Chinese meteria medica. Chin J new drugs 2011, 20(24):2424-2429.

[8] Xie ying, Wu jian-guo, Li wei et al: Toxicity prediction of unknown compounds based on gspan algorithm. Journal of Hefei university of technology. natural science edition 2007, 30(10):1278-1280.

[9] Wang jian-suo, Lai lu--hua, Tang you-qi:Prediction toxicology and database excavation of compounds. Chinese science bulletin 2000, 45(4):360-364.

[10] Wang bin, Yugang, Zhang zu-lin: Quantitative structure activity relationship and joint toxicity prediction of alkyl alcohos. Chinese science bulletin 2006, 51(13):1513-1518.

[11] Liu yang, Wang yon-hua, Li yen:calculation and prediction of environmental carcinogens. Journal of molecular science 2008, 24(6):393-398.

[12] Cao jia, Zheng yu-xin Zhou zon-can: Progress and hotspots in toxicology. China science foundation 2011(3):138-147.

[13] Li hon, Wang chon-jun,: Application of bioinformatics in the toxicogenomics research. Journal of Bioinformatics.2010, 8(4):330-333. 
[14] Dai yu-fei, Zheng yu-xin:Reexamination of the central principle of toxicology--doseresponse relationship of toxicant stimulation and it's impact on toxicology development. J of Foreign medical sciences(section of hygiene)2003, 30(4):246-249.
[15] John Paul Gosling: The importance of mathematical modelling in chemical risk assessment and the associated quantification of uncertainty. Computational toxicology 2019 10 44-50. 